\title{
Table of cited WTO and GATT dispute settlement reports
}

\section{GATT CASES}

EEC - Bananas II, GATT Panel Report, EEC - Import Regime for Bananas, DS38/R, 11 February 1994, unadopted 104

Jamaica - Margins of Preference, GATT Panel Report, Jamaica - Margins of Preference, L/3485, adopted 2 February 1971, BISD 18S, p. 183 ..... 101

Norway - Apples and Pears, GATT Panel Report, Norway - Restrictions on Imports of Apples and Pears, L/6474, adopted 22 June 1989, BISD 36S, p. 306

Spain - Soyabean Oil, GATT Panel Report, Spain - Measures Concerning

Domestic Sale of Soyabean Oil - Recourse to Article XXIII:2 by the

United States, L/5142, 17 June 1981, unadopted $101-102$

Thailand - Cigarettes, GATT Panel Report, Thailand - Restrictions on Importation of and Internal Taxes on Cigarettes, DS10/R, adopted 7 November 1990, BISD 37S, p. 200

Uruguay - Recourse to Article XXIII, GATT Panel Report, Uruguayan

Recourse to Article XXIII, L/1923, adopted 16 November 1962,

BISD 11S, p. 95 100,101

US - Manufacturing Clause, GATT Panel Report, United States Manufacturing Clause, L/5609, adopted 15 May 1984, BISD 31S, p. 74 102,103

\section{WTO CASES}

Argentina - Footwear (EC), Appellate Body Report, Argentina - Safeguard Measures on Imports of Footwear, WT/DS121/AB/R, adopted

12 January 2000, DSR 2000:I, p. 515 129

Brazil - Desiccated Coconut, Appellate Body Report, Brazil - Measures

Affecting Desiccated Coconut, WT/DS22/AB/R, adopted 20 March 1997, DSR 1997:I, p. 167

Brazil - Retreaded Tyres, Appellate Body Report, Brazil - Measures

Affecting Imports of Retreaded Tyres, WT/DS332/AB/R, adopted

17 December 2007, DSR 2007:IV, p. 1527 
Canada - Renewable Energy/Canada - Feed-In Tariff, Appellate Body

Reports, Canada - Certain Measures Affecting the Renewable

Energy Generation Sector/Canada - Measures Relating to the Feed-In

Tariff Program, WT/DS412/AB/R/WT/DS426/AB/R, adopted 24 May 2013, DSR 2013:I, p. 7

Canada - Renewable Energy/Canada - Feed-In Tariff, Panel Reports,

Canada - Certain Measures Affecting the Renewable Energy Generation

Sector/Canada - Measures Relating to the Feed-In Tariff Program,

WT/DS412/R and Add.1/WT/DS426/R and Add.1, adopted 24 May

2013, as modified by Appellate Body Reports WT/DS412/AB/R/WT/

DS426/AB/R, DSR 2013:I, p. 237

China - Auto Parts, Appellate Body Reports, China - Measures Affecting

Imports of Automobile Parts, WT/DS339/AB/R/WT/DS340/AB/R/

WT/DS342/AB/R, adopted 12 January 2009, DSR 2009:I, p. 3 ....... 5, 196

China - Auto Parts, Panel Reports, China - Measures Affecting Imports of

Automobile Parts, WT/DS339/R, Add.1 and Add.2/WT/DS340/R,

Add.1 and Add.2/WT/DS342/R, Add.1 and Add.2, adopted 12 January

2009, upheld (WT/DS339/R) and as modified (WT/DS340/R/WT/

DS342/R) by Appellate Body Reports WT/DS339/AB/R/WT/

DS340/AB/R/WT/DS342/AB/R, DSR 2009:I, p. 119 $112,114,120$

China - Electronic Payment Services, Panel Report, China - Certain

Measures Affecting Electronic Payment Services, WT/DS413/R and

Add.1, adopted 31 August 2012, DSR 2012:X, p. 5305 5

China - Publications and Audiovisual Products, Appellate Body Report, China

- Measures Affecting Trading Rights and Distribution Services for

Certain Publications and Audiovisual Entertainment Products,

WT/DS363/AB/R, adopted 19 January 2010, DSR 2010:I, p. 3 ........ 5, 99,

114, 120, 141, 149, 169, 170, 171, 173, 174, 184, 185, 186, 188

China - Publications and Audiovisual Products, Panel Report, China -

Measures Affecting Trading Rights and Distribution Services for

Certain Publications and Audiovisual Entertainment Products,

WT/DS363/R and Corr.1, adopted 19 January 2010, as modified

by Appellate Body Report WT/DS363/AB/R, DSR 2010:II, p. 261 64

$113,114,120,168,169$,

185,186

China - Rare Earths, Appellate Body Reports, China - Measures Related

to the Exportation of Rare Earths, Tungsten, and Molybdenum,

WT/DS431/AB/R/WT/DS432/AB/R/WT/DS433/AB/R, adopted

29 August 2014 ................ 5, 57-58, 58, 59, 115, 122, 127, 129, 130, 135,

179-187, 203, 212, 276

China - Rare Earths, Panel Reports, China - Measures Related to the

Exportation of Rare Earths, Tungsten, and Molybdenum, WT/DS431/R

and Add.1/WT/DS432/R and Add.1/WT/DS433/R and Add.1, adopted

29 August 2014, upheld by Appellate Body Reports

WT/DS431/AB/R/WT/DS432/AB/R/WT/DS433/AB/R 122, 176 , 
China - Raw Materials, Appellate Body Reports, China - Measures Related to the Exportation of Various Raw Materials, WT/DS394/AB/R/WT/DS395/ AB/R/WT/DS398/AB/R, adopted 22 February 2012, DSR 2012:VII, p. 3295 ....................... 5, 99, 143, 144, 174, 176, 178, 186, 212, 276, 284

China - Raw Materials, Panel Reports, China - Measures Related to the Exportation of Various Raw Materials, WT/DS394/R, Add.1 and Corr.1/WT/DS395/R, Add.1 and Corr.1/WT/DS398/R, Add.1 and Corr.1, adopted 22 February 2012, as modified by Appellate Body Reports WT/DS394/AB/R/WT/DS395/AB/R/WT/DS398/AB/R, DSR 2012:VII, p. 3501 ........ 64-65, 80, 114, 115, 144, 145, 150, 173, 174, 176, 186, 218,

Colombia - Textiles, Panel Report, Colombia - Measures Relating to the Importation of Textiles, Apparel and Footwear, WT/DS461/R and Add.1, circulated to WTO Members 27 November 2015, p. 141

EC - Asbestos, Panel Report, European Communities - Measures Affecting Asbestos and Asbestos Containing Products, WT/DS135/R and Add. 1, adopted 5 April 2001, as modified by Appellate Body Report WT/DS135/AB/R

EC - Bananas III (Article 21.5 - Ecuador II)/EC - Bananas III

(Article 21.5 - US), Appellate Body Reports, European Communities Regime for the Importation, Sale and Distribution of Bananas - Second Recourse to Article 21.5 of the DSU by Ecuador, WT/DS27/AB/RW2/ ECU, adopted 11 December 2008, and Corr.1/European Communities Regime for the Importation, Sale and Distribution of Bananas Recourse to Article 21.5 of the DSU by the United States, WT/DS27/ AB/RW/USA and Corr.1, adopted 22 December 2008, DSR 2008:XVIII, p. 7165

EC - Chicken Cuts, Appellate Body Report, European Communities Customs Classification of Frozen Boneless Chicken Cuts, WT/DS269/ AB/R/WT/DS286/AB/R, adopted 27 September 2005, and Corr.1, DSR 2005:XIX, 9157

EC - Fasteners (China), Appellate Body Report, European Communities Definitive Anti-Dumping Measures on Certain Iron or Steel Fasteners from China, WT/DS397/AB/R, adopted 28 July 2011, DSR 2011:VII, p. 3995 255-256, 257

EC - Fasteners (China), Panel Report, European Communities - Definitive Anti-Dumping Measures on Certain Iron or Steel Fasteners from China, WT/DS397/R and Corr.1, adopted 28 July 2011, as modified by Appellate Body Report WT/DS397/AB/R, DSR 2011:VIII, p. 4289

EC - Fasteners (China) (21.5), Appellate Body Report, European Communities - Definitive Anti-Dumping Measures on Certain Iron or Steel Fasteners from China - Recourse to Article 21.5 of the DSU by China, WT/DS397/AB/RW and Add.1, adopted 12 February 2016

EC - Seal Products, Appellate Body Reports, European Communities Measures Prohibiting the Importation and Marketing of Seal Products, WT/DS400/AB/R/WT/DS401/AB/R, adopted 18 June 2014 140,141 
EU - Biodiesel, Appellate Body Report, European Union - Anti-Dumping Measures on Biodiesel from Argentina, WT/DS473/AB/R, adopted 26 October 2016

EU - Biodiesel, Panel Report, European Union - Anti-Dumping Measures on Biodiesel from Argentina, WT/DS473/R, adopted 26 October 2016, as modified by Appellate Body Report WT/DS473/AB/R

EU - Footwear, Panel Report, European Union - Anti-Dumping Measures on Certain Footwear from China, WT/DS405/R, adopted 22 February 2012, DSR 2012:IX, p. 4585 $5,117,258$

Guatemala - Cement I, Appellate Body Report, Guatemala - Anti-Dumping Investigation Regarding Portland Cement from Mexico, WT/DS60/AB/R, adopted 25 November 1998, DSR 1998:IX, p. 3767

Japan - Alcoholic Beverages II, Appellate Body Report, Japan - Taxes on Alcoholic Beverages, WT/DS8/AB/R/WT/DS10/AB/R, WT/DS11/AB/R, adopted 1 November 1996, DSR 1996:I, p. 97

Peru - Agricultural Products, Appellate Body Report, Peru - Additional Duty on Imports of Certain Agricultural Products, WT/DS457/AB/R and Add.1, adopted 31 July 2015

Ukraine, Armenia - Measures Affecting the Importation and Internal Sale of Cigarettes and Alcohol Beverages, WT/DS411/2/Rev.1, 8 October 2010

US - Anti-Dumping and Countervailing Duties (China), Appellate Body Report, United States - Definitive Anti-Dumping and Countervailing Duties on Certain Products from China, WT/DS379/AB/R, adopted 25 March 2011, DSR 2011:V, p. 2869 $5,50,127$

US - Anti-Dumping and Countervailing Duties (China), Panel Report, United States - Definitive Anti-Dumping and Countervailing Duties on Certain Products from China, WT/DS379/R, adopted 25 March 2011, as modified by Appellate Body Report WT/DS379/AB/R, DSR 2011:VI, p. 3143

US - Carbon Steel, Panel Report, United States - Countervailing Duties on Certain Corrosion-Resistant Carbon Steel Flat Products from Germany, WT/DS213/R and Corr.1, adopted 19 December 2002, as modified by Appellate Body Report WT/DS213/AB/R, DSR 2002:IX, p. 3833

US - Clove Cigarettes, Appellate Body Report, United States - Measures Affecting the Production and Sale of Clove Cigarettes, WT/DS406/ AB/R, adopted 24 April 2012, DSR 2012: XI, p. 5751

US - Clove Cigarettes, Panel Report, United States - Measures Affecting the Production and Sale of Clove Cigarettes, WT/DS406/R, adopted 24 April 2012, as modified by Appellate Body Report WT/DS406/AB/R, DSR 2012: XI, p. 5865

US - COOL, Panel Reports, United States - Certain Country of Origin Labelling (COOL) Requirements, WT/DS384/R/WT/DS386/R, adopted 23 July 2012, as modified by Appellate Body Reports WT/DS384/AB/ R/WT/DS386/AB/R, DSR 2012:VI, p. 2745 
US - Countervailing and Anti-Dumping Measures (China), Appellate Body Report, United States - Countervailing and Anti-Dumping Measures on Certain Products from China, WT/DS449/AB/R and Corr.1, adopted 22 July 2014, DSR 2014:VIII, p. 3027

US - Gasoline, Appellate Body Report, United States - Standards for Reformulated and Conventional Gasoline, WT/DS2/AB/R, adopted 20 May 1996, DSR 1996:I, p. 3 $1,109,140,141,145-146$

US - Shrimp, Appellate Body Report, United States - Import Prohibition of Certain Shrimp and Shrimp Products, WT/DS58/AB/R, adopted 6 November 1998, DSR 1998:VII, p. 2755 143,145

US - Shrimp II (Vietnam), Appellate Body Report, United States Anti-Dumping Measures on Certain Shrimp from Viet Nam, WT/DS429/AB/R, adopted 22 April 2015, and Corr.1

US - Shrimp II (Vietnam), Panel Report, United States - Anti-Dumping Measures on Certain Shrimp from Viet Nam, WT/DS429/R and Add.1, adopted 22 April 2015, upheld by Appellate Body Report WT/DS429/AB/R $118,150,265,266,299-300$

US - Shrimp (Viet Nam), Panel Report, United States - Anti-Dumping Measures on Certain Shrimp from Viet Nam, WT/DS404/R, adopted 2 September 2011, DSR 2011:X, p. 5301

US - Tuna II (Mexico), Appellate Body Report, United States - Measures Concerning the Importation, Marketing and Sale of Tuna and Tuna Products, WT/DS381/AB/R, adopted 13 June 2012

US - Tuna II (Mexico), Panel Report, United States - Measures Concerning the Importation, Marketing and Sale of Tuna and Tuna Products, WT/DS381/R, adopted 13 June 2012, modified by Appellate Body Report WT/DS381/AB/R

US - Tyres (China), Appellate Body Report, United States - Measures Affecting Imports of Certain Passenger Vehicle and Light Truck Tyres from China, WT/DS399/AB/R, adopted 5 October 2011, DSR 2011:IX, p. 4811 $5,192,193,244$

US - Tyres (China), Panel Report, United States - Measures Affecting Imports of Certain Passenger Vehicle and Light Truck Tyres from China, WT/DS399/R, adopted 5 October 2011, upheld by Appellate Body Report WT/DS399/AB/R, DSR 2011:IX, p. 4945 ........ 191-192, 244, 